\title{
Victor-Alexandru Briciu
}

Transilvania University of Brasov, Brasov, Romania

e-mail: victor.briciu@unitbv.ro

\section{Arabela Briciu}

Transilvania University of Brasov, Brasov, Romania

\section{Florin Nechita}

Transilvania University of Brasov, Brasov, Romania

\section{Content Analysis Made Simple for Students. An Interactive Online Application Solution}

\begin{abstract}
This paper aims to present an interactive online solution developed for students to better understand the application of content analysis (as a research method) and the use of a coding scheme (as the research instrument) by using a software platform, an application for analysing an online place brand's content, in terms of the characteristics of a place brand's identity communicated through official websites.
\end{abstract}

Keywords: content analysis, online environment, application, software platform

\section{Introduction. Defining the Cyberspace}

The term cyberspace was first introduced into the English language by Williams Gibson in his science fiction novel, Neuromancer, in 1984. According to the author, cyberspace represents a "consensual hallucination experienced daily by billions of legitimate operators, in every nation, by children being taught mathematical concepts... A graphic representation 
of data abstracted from the banks of every computer in the human system" (Cotton and Oliver 1994 apud Bryant 2001, p. 139). However the present connotation of the word has changed significantly.

According to the Cambridge Dictionary, cyberspace refers to the "Internet considered as an imaginary area without limits where you can meet people and discover information about any subject: You can find the answer to almost any question in cyberspace" (Cambridge Dictionary, dictionary.cambridge.org/dictionary/english/cyberspace, accessed on 24.12.2017). Although this definition is not completely accurate, it does point out several characteristics of cyberspace: its non-physicality, its social dimension and its functionality. Vande Moere claims that the term cyberspace is technically unimportant since there are other phrases used synonymously: the Internet, the digital domain, computer networking, data space, the informational sphere etc. However this form connotes automation, artificial control and computerization as well as a multidimensional place (Vande Moere 1998, p. 5).

Rebecca Bryant defines cyberspace as "the new medium of communication, electronic communication, which is fast outmoding, or even replacing more traditional methods of communication" (Bryant 2001, p. 139). David Holmes claims that cyberspace is "a global space inhabited everyday" (Holmes 2001, p. 6).

The origins of the Internet, or cyberspace as you wish you call it, are to be found in a military research project created by the Defense Department of United States (Briciu et al 2019a, p. 149). In 1958, ARPA (Advanced Research Projects Agency) was created to mobilize research resources, particularly from the university world, in order to create military superiority over the Soviet Union. In September 1969 a minor project, a computer network, emerging from one of ARPA'S department called ARPANET was invented. Its purpose was to facilitate "a way of sharing computing time on-line between various computers centres and research groups working for the agency" (Castells 2001, p. 10).

Before 1990, cyberspace was used primarily for e-mails and filetransfers by academics and researchers across the United States of America and was defined by the shared pursuit of science. Nonetheless, by the middle of the decade two major changes occurred: the commercial use (once forbidden across the US) surpassed its academic use and the World 
Wide Web (WWW), a graphical-hypertextual interface, became the main vehicle for navigating internet sites (Holmes 2001, p. 57).

The expansion and usage of the cyberspace has grown very rapidly in the last years and the number of other media: e-mail, mailing lists, chat rooms and virtual worlds has expended greatly and are used by a rapidly expanding population. The particular characteristics of cyberspace are represented by its space, that it can only be entered by specially designed devices and that it enables communication between individuals mostly independent of time and space (Vande Moere 1998, p. 11).

The first distinctive feature of the cyberspace is its spatiality. $\mathrm{Nu}$ merous people consider that the space can be either physical either nonphysical (in our case virtual). Despite this general belief, specialists in the filed try their very best to explain why cyberspace cannot be perceived as being only virtual or only physical. Their conclusion is that cyberspace is only partially physical therefor it represents a different kind of space.

In the ordinary language, the physical space is determined by its relationship with the human body because it represents the space in which the human bodies move and interact with one another. The physical space is a space "for the body, perceived and occupied by the body and in many respects directing the movements of the body" (Saco 2002, p. 25). By extension, virtual is only non-physical because it is something unopened to unmediated perception. The term physical, in general, is understood as a phenomenon that can be perceived by sight, taste, touch, smell and hearing. However this understanding of physical is utterly wrong considering the numerous phenomena of the physical existence that cannot be directly perceived by the human body such as gravity.

From a conventional point of view, virtual represents only a nonphysical space and it is understood as anything that is in digital form: "combinations of binary programming codes and electrical impulses that make up the bulk of the invisible data traversing computers circuits and network routes" (Saco 2002, p. 25). While these data may include simulations of something that conventionally is physical, it can only exist in a digital form therefor virtual can only be non-physical. From a technical point of view, virtual refers to "a digital representation of something else, typically something that does or will exist in the physical world" (Saco 2002, p. 24). 
Regarding the issue of space, Rebecca Bryant says that we should have in mind two aspects of the cyberspace. "On the one hand, we have virtual reality - a 3-D cyberspatial environment which humans can "enter" and "move things", interacting with both the computer and other human beings. One the other hand, we have the slightly less dramatic, but more utilitarian, world of networks of computers linked via cables and routers (similar to telephone connections) which enable us to communicate, store and retrieve information" (Bryant 2001, pp. 139-140). Vande Moere states that cyberspace it is a virtual place that is both real and artificial at the same time therefor by definition not a physical location and it can be considered as a "digital complement of our atomic world" (Vande Moere 1998, p. 11). The biggest difference between cyberspace and physical space is that it involves an electromagnetic force rather than a mechanical one. As a result of this it has different physical proprieties.

Cyberspace has a geography of its own, made of networks and nodes in which process information travels from the places where they are generated and managed. "The resulting space of flows is a new form of space, characteristic of the Information Age, but it is not placeless: it links places by telecommunicated computer networks and computerized transportation systems. It redefines distance but does not cancel geography" (Castells 2001, p. 207). Cyberspace has become a global space that is inhabited everyday by a large number of people.

As mentioned earlier, the second characteristic of the cyberspace is that it can only be entered through special physical access devices "with an artificial processing mechanism, such as digital computing power and/or software that is joined with other access devices on a network of physical connection... without an access device, there is no distinction between cyberspace and communications in the real world" (Vande Moere 1998, p. 11).

The virtual space can be entered only through a physical interface such as "personal computers, monitors, keyboards, pointing devices, microphones, video cameras, modems, network interface cards, servers, routers, switches, telephone lines, coaxial cables, fiber-optic cables, radio towers, communications satellites, and, increasingly, handheld computers and Internet-ready cell phones" (Saco 2002, p. 26). Moreover, these are not the only physical components that represent the minimum condition necessary for the existence of the virtual space. Currents of binary data 
such as electricity, pulses of light and microwaves that flow in and out of them and form computer networks are themselves physical.

Last but not least, the third characteristic of the cyberspace is its social dimension. To be noted that originally the computer was designed and used for its capacity of a processor. However, in the 1990's, when the fascination with information technology occurred, the computer started to function as a "communication and social environment, rather than its capacity as a processor" (Holmes 2001, p. 58). Without this element, now it is impossible to understand the Internet. In fact, Rebecca Bryant states that cyberspace represents "the new medium of communication, electronic communication, which is fast outmoding, or even replacing, more traditional methods of communication" (Bryant 2001, p. 139).

Once it became the new medium of communication, cyberspace had to combat claims regarding the rising of new patterns of social interaction. Manuel Castells responds to these claims saying that "social interaction on the Internet does not seem to have a different effect on the patterning of everyday lifestyle, generally speaking, except for adding on-line interaction to existing social relationships" (Castells 2001, p. 119). Diana Saco states that "networking practices and orders are related as well to a variety of lived practices through which the spatiality of cyberspace is culturally experienced" (Saco 2002, p. 28). There are numerous practices online that represent extensions of face to face practices such as: homepages on websites that signify an arrival somewhere, participating in forums that signify being part of a community, e-mail "courtship" and so on.

Cyberspace enables interaction and communication between individuals mostly independent of time and space. "The sense of immediacy that apparently results from interactions in cyberspace is in fact artificial at best, since these human communications almost always lack similarity of place, and usually also happen in a shifted and different time" (Vande Moere 1998, p. 11). In contrast to normal interactions, cyberspace interactions are usually indirect, delayed in time or separated by distance.

In order to understand better the new forms of social interaction on cyberspace is to "build on a definition of community, de-emphasizing its cultural component, emphasizing its supportive role to individuals and families, and de-linking its social existence from a single kind of material support" (Castells 2001, p. 127). In sociology, communities are based on the sharing of values and social organization. In this new context they 
represent "networks of interpersonal ties that provide sociability, support, information, a sense of belonging, and social identity" (Wellman, 2001 apud Castells 2001, p. 127).

The Internet has an enormous impact on a wide ranging set of practices. These include changes in "interpersonal communication, sociality, and community: in courtship and romantic relationships and even in divorce; in surveillance and privacy issues; in grassroots social movements and campaign politics; in education, literature, authorship, and readership; in class politics; in statecraft; and most profoundly in potential changes to the construction and presentation of self" (Saco 2002, p. 9).

In conclusion, the term cyberspace first appeared in the English language in a since fiction novel. It represents a virtual place that is both real and artificial at the same time, a new medium of communication, a global space inhabited every day. The origins of cyberspace are to be found in a military research program designed to share computing time between various computers. Throughout time, numerous changes took place regarding the cyberspace. Two of the biggest changes appeared in 1990's when the commercial use surpassed the academic use and the World Wide Web (www) became the main vehicle for navigating Internet sites. Since then, the usage of cyberspace has grown very rapidly and currently more than $50 \%$ of world population uses it. There are three main characteristics of cyberspace: its spatially, its social component and that it can only be entered through a physical interface. Cyberspace is only partially physical therefor it represents a different kind of space and it is a digital complement to our atomic world. It can only be accessed through specially designed devices and although originally was designed to be used for its capacity as a processor, now it has become a communication and social environment.

\section{Using the Content Analysis Method and the Research Objective}

Although content analysis in general, and its application to media sources, in particular, has a rich theoretical and methodological content, "Web site analysis is a recent study framework", considers Gibson and Ward (2000, p. 303). Applicability in the online environment is supported by 
the explanation that content analysis would be "an observational research method used to systematically evaluate the symbolic content of all recorded forms of communication" (Kolbe and Burnett 1991, p. 243). Considering content analysis a method of social field research without affecting it in any way, Babbie (2010) classifies it into a broader framework, as an observational method, as a form of discrete and nonreactive research (Webb et al. [1966] 2000 apud Babbie 2010, pp. 437-438) and representing "the study of recorded human communications, such as books, websites, pictures and laws" (Babbie 2010, p. 438).

However, not all scientific perspectives consider such a position, as evidenced by Heath, who believes that "although some question whether content analysis is a qualitative or a quantitative method, its use in public relations is most often seen as qualitative or informal" (2005, p. 727). The same author returns to the problem in another paragraph of the paper, explaining that "It approaches a quantitative, formal method in that certain rules for its conduct are established beforehand and the messages are then analyzed according to those rules. Because it works on messages that have already been uttered, written, presented, or published, it may fit better as a qualitative method; when used as a measurement instrument, however, it fits better as a quantitative method" (Heath, 2005, p. 727). According to Chelcea (2004, p. 543), the most acceptable definition of content analysis is proposed by Holsti. It states that: "Content analysis is that research technique that allows inferences by systematically and objectively identifying specific characteristics within a text" (Holsti 1968, p. 601). Also, Chelcea has imposed a number of clarifications on this definition. "Being a research technique, content analysis must be integrated into a methodology, in accordance with one theory or another" (2004, p. 544).

Finally, Iluţ presents a more general definition, according to which content analysis is "a method that refers to the quantitative analysis of documents, with the aim of highlighting themes, tendencies, attitudes, values or patterns of association of themes and evaluations (of attitudes and values). It has also been called the quantitative treatment of qualitative symbolic material" (1997, p. 135).

In this article, we agree with the ideas presented above regarding the determining role of content analysis, as a method for examining the manifest content of messages, while also being an ideal tool for examin- 
ing websites. In addition, we must accept that "content analysis is traditionally considered, first and foremost, a quantitative method (Berelson 1952 Stern 1989), but which also requires a qualitative evaluation" (apud Hwang et al. 2003, p. 13). In the light of these last clarifications, we will consider the content analysis as a research method, being at the border between the two research models, as a quantitative-qualitative variant of analysing the content of communication, the frequency analysis the research technique and the grid of categories - the research instrument for coding and quantitative processing the collected data and the adaptation by using a software platform, an application for analysing an online place brand's content, in terms of the characteristics of a place brand's identity communicated through official websites.

For testing the new software application we have established how each indicator will receive a value, a code, a score. At this stage we reviewed the criteria established by the other authors in their own analysis grids (Gibson and Ward 2000, Schneider and Foot 2002, Florek et al. 2006, Brundin 2008, Okazaki and Škapa 2009), adapting these values to the specificity and objective of our own research approach (ie, to analyze the characteristics of a place brand's identity communicated through official websites), as this methodological process is explained by Hwang et al. (2013): "Researchers generally need to develop their own coding scheme for analyzing content, although sometimes researchers can adopt existing coding schemes established by their peers. Human intuition can intervene in the process of developing coding schemes. Although all research is subject to some human intuition, the process of content analysis allows relatively more room for the researcher's intuition than do some methods such as surveys or experimental designs"(p. 13).

We followed the methodological rigor set out by Rotariu (1991), who considered that the respective grid and categories should be: "exhaustive (to surprise all the variants of appearance of the features of the theme); exclusive (one unit should be registered in only one category); objective (to allow a classification of the material that depends as little as possible on the analyst who performs it), and relevant (appropriate to the objective pursued by the research and the content of the documents)" (apud Iluț 1997, p. 137).

The next step, by applying the research instrument to the sites selected in the analysis and defining them through the software application, 
is to give values to the indicators, based on the coding scheme established, following the same procedure, to ensure repeatability and internal consistency. All values were manually encoded and recorded in the defined fields of the database within the software application.

\section{Presenting the Application for Content Analysis}

The application was implemented using up to date programming languages and technologies. For the database implementation, MySQL was used together with phpMyAdmin tool (Briciu et al 2019c, p. 9). The application was developed using PHP, CSS and JavaScript being used for designing it. This application is not designed for general or public use yet, it is intended to analyse the brand characteristics added by a user (Briciu et al 2019b, p. 759).

Once created, a user can add the brands needed for the analysis (i.e., to identify and analyze the characteristics of place brand online identity), according to the analysis grid or the coding scheme used on a quantitative research level, by applying the content analysis method to the official sites of the place brands. Under this procedure, the analysis unit was considered "the page" of the official websites of the place brands. The starting point in applying the procedure for establishing the identity characteristics according to the coding scheme, is the site Homepage, because sites of different sizes can be compared in this manner, using the same degree of size, as a measure to "equalize differences between small sites with few links and larger sites with many links" (Hwang et al. 2003, p. 14).

This grid was defined "with a very simple interface to be used by anyone. Buttons or text fields were created for the input of the variables, the user only having to enter the data according to the research" (Briciu et al 2019a, p. 151). Once a brand is added by the user, the score is calculated according to its characteristics. This score is calculated for all four dimensions that need to be analysed (i.e., (1) Brand design or the brand visual presentation, (2) Brand behaviour, (3) Brand information and communication and (4) Site delivery or Exposure of brand features). Depending on the score obtained, the brand is added to the diagram of each dimension and to the position obtained. After several brands have been added, the user can analyse the charts, obtaining the necessary in- 
formation needed. The database was created by using the phpMyAdmin tool that is included xampp. It has a modern visual interface. Every entry in the database has a unique ID that is auto incremented. The application data was dived into three categories: Admin, User and Site.

The in-app login is per session. "A session is the time period when multiple PHP scripts, accessed at different times that can store and use shared information" (Briciu et al 2019a, p. 152). A session starts when a script calls the session_start function and ends when the user closes the browser. The code is made to generate a single admin key that is stored in the database in the session_id field, and is also stored in a session variable. At each request of a page, it is verified that the session_id in the logged-in database is identical to the session_id that exists in the session variable, so if the admin logs on Firefox, and after logging on to Chrome, the first request made on Firefox will be sign out and asked to re-log because the session has expired.

The admin section is dedicated exclusively to the application manager. In this section a regular user does not have the right to enter or log in. The administration account of the application can only be created from the database by its administrator. The application administrator can add users, and once a user has been added, that account can be disabled by a simple click, if necessary. The administrator also has the right to change the data from an already created account.

By clicking on a user's account from the list of all users, the administrator can see all the brands (sites) he added, also having the right to approve or reject a user-added brand. It is worth mentioning that a brand that is not approved is not taken into account in the analysis. As mentioned above, the administrator has the right to add users. Being an application for a particular research purpose, not for the general public usage, it was agreed that not anyone who accesses the app should be able to create an account.

Once the user account has been created by the application administrator, it can log in using the login form. After logging in, the user has three pages to use as follows: Dashboard, My Account and My Sites. The "Dashboard" page is the app's home page where the user can see the analysis charts for the brands he added. These are calculated based on the presence or absence of certain attributes of the brand. 
On the "My Account" page, users can update the data, so they can change their fields containing their email address, First Name, Last Name and phone number. Also, the password change function by the user was implemented on this page, without involving the administrator in this task as well. The "My Sites" section of the menu allows the user to add brands to be analyzed. This page is made up of several data entry forms created after the analysis grid used. Also, on this page, the user can see all the sites he added, which can be modified as needed.

In this section of the user's options, he or she can add a site for analysis. The content of this section is more complex because it is composed of several forms, according to the analysis chart resulting from the initial research. The branding page (Add new site) is composed of two general parts: the generic brand information form (name, URL, country, etc.) and forms for entering the variables of the four dimensions analyzed: Brand design, Brand behaviour, Brand communication and Site delivery/ Exposure of brand features.

The user can easily enter the variables using this form, since it was created for easy use with a user-friendly interface. For example, if a brand has the "Slogan" variable in its composition, the user can express it by clicking the button next to the "Slogan" variable. The button initially has the value "0", meaning "No" (Briciu et al 2019b, p.759). Once accessed, the button switches to " 1 ", meaning "Yes". Note that within the forms in this section of the application, users will still be able to enter the absolute values of the variables, the only difference being considered in how the score will be calculated. This result has been reached because the values of the total scores of each dimension should be as close as possible, and if, for example, a site would use a large number of cookies, the value of the variable would also be very high, and compared to the other dimensions, a much higher score would be obtained, without any justification. For this reason, the choice has been made to weigh the variables that could have high values, reaching their simple dichotomous status of presence (0), or absence (1).

Returning to the functionality of adding brand values along with all of identity variables, after the user has finished setting all variables based on brand values, in order for the site to be added to the database, you must press the "Add Site" button. This will trigger the addSite () function that will make the transition to MySQL. This function has the general 
configuration as any other add-on function, but since it adds a very large number of variables, it is very long.

Once added, the site will appear in the list of sites added by that user. This list has a similar interface to the list of all users in the Admin section, and consists of the following fields: the brand ID, Name, URL, Country, Continent, Date it was added, Brand design dimension score, Brand behaviour dimension score, Brand communication dimension score, Site delivery/ Exposure of brand features dimension score, Status (Active or Inactive) and Actions (Explore or Delete) (Briciu et al 2019c, p. 10). The list also allows you to sort brands according to each field, if the number added is large, it is possible to display only one part of the total number of brand records on a page, so you can navigate between the pages in the "Previous" and "Next". The search function is also present in this list, searching in real-time, without the need for a page reload.

If users want to change certain variables or fields of the brand added, they can modify this by either clicking on the brand name or the icon on the Action bar. This action will open a new form of the brand, containing all fields filled in. The user can make the desired changes, and then clicking the Update site info button will update the site's data. This is done with the updateSite() function. Once added, a brand must first be approved by the administrator. Once it is approved, it will be available so that it can be taken into account when calculating diagrams. The administrator may also disable a site at any time if it is not in accordance with the analysis procedure.

The Dashboard page lists the rankings of each dimension, depending on the scores of the brands added or considered by the user. A brand can get a maximum score within the brand construction dimension, but it can also score with a lower score in another dimension. By using this chart, the user will be able to continue the initial research.

The general interface was designed using the Bootstrap module (Briciu et al 2019b, p. 759) and uses the latest updates of the used technologies. CSS and jQuery were used for stylization. Within the general interface, you can find the menu and navigation bar, and the user's homepage is the Dashboard page, which contains the analysis charts.

"The button that shows two arrows to the left is to narrow the menu if necessary or if this is preferred. Also, this is helpful if the application runs at a lower resolution. If you opt for this style, the menu will become 
a narrow bar where only the icons of each category will be displayed, and when the mouse pointer is over the icons, a text with the name of that category appears from the menu. It should be noted that this happens automatically if the application detects a very low resolution, without requiring the user to intervene" (Briciu et al 2019a, p. 159).

It can be mentioned that within the site, the section dedicated to the user account is presented now. Instead of the user account, the user name and surname that the user provided to the application manager are displayed (in this case: User Name). If the user accesses the category of adding a new brand or exploring an already defined brand, the navigation bar will have the following configuration.

\section{Conclusions}

This application was created to digitize the data entry process and automate the creation of the diagrams needed for the research analysis. At the foundation of the application is the PHP language, in addition to the other technologies used which also contribute a lot to the development of the application.

The purpose of this article was to present the content analysis made simple for students by implementing a software platform to facilitate the analysis of the identity components specific to the local brands in the online environment, this goal being achieved by implementing all the functions that perform data collection, calculation of scores, but also displaying them in diagrams. All variables were defined and named according to the analysis grid elaborated before.

Using this software, students can enter the data obtained by simply accessing the associated user account. Thus, the time spent calculating scores and making diagrams for analysis is greatly reduced.

As further development possibilities, the application can be associated with a domain and hosted in the online environment, being accessible at any time or an installation kit can be created, with the help of which the software can be installed on any computer, without the need for Internet access.

Another development possibility is the creation of an option to select the brand category, so that charts can be sorted and made only for the brands (or any other research subject) in the desired category. 


\section{Acknowledgement}

This work was supported by a grant of the Romanian National Authority for Scientific Research and Innovation, CCCDI - UEFISCDI, project number ERANET-FLAG - FuturICT2.0, within PNCDI III.

\section{References}

Babbie, E. (2010), The practice of social research, Iasi: Polirom Publishing House. Briciu, V.-A., Briciu, A. \& Demeter, R. (2019a), A Tentative Model for an Online Place Branding Application Solution, "Bulletin of the Transilvania University of Brasov", 12(61), p. 149-160, https://doi.org/10.31926/but.ssl.2019.12.61.1.14.

Briciu, V.-A., Demeter, R., Nechita, F., Kavoura, A. \& Briciu, A. (2019b), A Proposed Online Platform for Ranking Place Brands Identity Characteristics of Official Tourism Websites [in:] Strategic Innovative Marketing and Tourism. Springer Proceedings in Business and Economics, ed. A. Kavoura, E. Kefallonitis \& A. Giovanis, Cham: Springer Nature Switzerland, https://doi. org/10.1007/978-3-030-12453-3_87.

Briciu, V.-A., Nechita, F., Demeter, R. \& Kavoura, A. (2019c), Minding the Gap Between Perceived and Projected Destination Image by Using Information and Communication Platforms and Software, "International Journal of Computational Methods in Heritage Science”, 3(2), p. 1-17, doi: 10.4018/ IJCMHS.2019070101.

Brundin, P. (2008), Politics on the Net. NGO practices and experiences, Örebro University Publisher.

Bryant, R. (2001), What Kind Of Space Is Cyberspace?, "Minerva - An Internet Journal of Phylosophy", 5, p. 138-155.

Castells, M. (2001), The Internet Galaxy. Reflections on the Internet, Business, and Society, New York: Oxford University Press.

Chelcea, S. (2004), Methodology of sociological research. Quantitative and qualitative methods, 2nd ed., Bucharest: Economic Publishing House.

Florek, M., Insch, A. \& Gnoth, J. (2006), City Council websites as a means of place brand identity communication, "Place Branding", 2(4), p. 276-296, doi: 10.1057/palgrave.pb.6000036.

Gibson, R. \& Ward, S. (2000), A Proposed Methodology for Studying the Function and Effectiveness of Party and Candidate Web Sites, "Social Science Computer Review", 18(3), p. 301-319, doi: 10.1177/089443930001800306. 
Heath, R. L. (2005), Encyclopedia of Public Relations, London: Sage Publications Inc.

Holmes, D. (2001), Virtual Globalization: Virtual Spaces/Tourists Spaces, London: Routledge.

Holsti, O. R. (1968), Content Analysis [in:] The Handbook of Social Psychology, 2nd ed., vol. II, ed. G. Lindzey \& E. Aronson, New Delhi: Amerind Publishing Co.

Hwang, J.-S., Mcmillan, S. \& Lee, G. (2003), Corporate web sites as advertising: an analysis of function, audience, and message strategy, "Journal of Interactive Advertising", 3(2), p. 10-23, doi: 10.1080/15252019.2003.10722070.

Iluţ, P. (1997), The qualitative approach of the sociouman, Iasi: Polirom Publishing House.

Kolbe, R. H. \& Burnett, M. S. (1991), Content-Analysis Research: An Examination of Applications with Directives for Improving Research Reliability and Objectivity, "Journal of Consumer Research", 18(2), p. 243-250.

Okazaki, S. \& Škapa, R. (2009), Understanding Brand Website Positioning in the New EU Member States: The Case of the Czech Republic [in:] Contemporary Research in E-Branding, ed. S. Bandyopadhyay, Hershey: Information Science Reference.

Saco, D. (2002), Cybering Democracy: Public Space and the Internet, Minneapolis: University of Minnesota Press.

Schneider, S. M. \& Foot, K. A. (2002), Online structure for political action: exploring Presidential campaign web sites from the 2000 American election, "The Public", 9(2), p. 1-18.

Vande Moere, A. (1998), The Language of Cyberspace: an Architectural Approach, graduate's thesis, K.U. Leuven University, Dept. of Architecture, Urban Design and Planning, Leuven, Belgium.

\section{Internet sources}

Cambridge Dictionary, https://dictionary.cambridge.org/dictionary/english/ cyberspace [access date: 24.12.2017]. 
